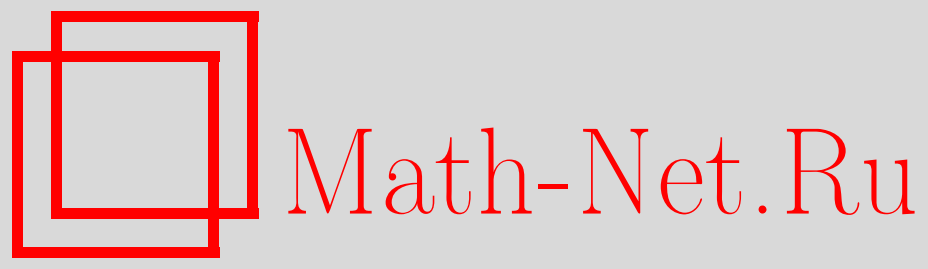

Г. И. Ольшанский, Разностные операторы и детерминантные точечные процессы, Функи. анализ и его прил., 2008, том 42, выпуск 4, 83-97

DOI: https://doi.org/10.4213/faa2932

Использование Общероссийского математического портала MathNet.Ru подразумевает, что вы прочитали и согласны с пользовательским соглашением

http://www. mathnet.ru/rus/agreement

Параметры загрузки:

IP : 34.229 .108 .108

26 апреля 2023 г., 03:20:25

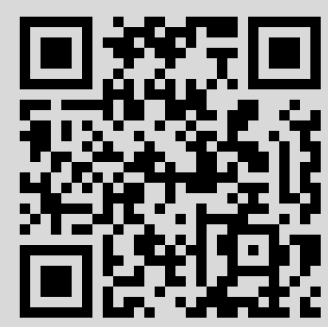


Функиионалъный анализ и его приложения

2008, т. 42, вып. 4, с. 83-97

УДК 519.218.5

\title{
Разностные операторы и детерминантные точечные процессы*
}

\author{
(c) 2008. Г. И. ОльшАНСКИй
}

Посвящается И. М. Гельфанду в связи с его 95-летием

\section{Введение}

Точечный процесс - это ансамбль случайных локально конечных конфигураций точек в некотором пространстве $\mathfrak{X}$. Удобным инструментом для работы с точечными процессами являются корреляционные функции (они аналогичны моментам случайных величин [19]). Корреляционные функции нумеруются натуральными числами $n=1,2, \ldots$, и $n$-я функция зависит от $n$ переменных, пробегающих $\mathfrak{X}$. Точечный процесс называется детерминантным ([5], [26]), если его корреляционные функции всех порядков могут быть представлены в виде главных миноров некоторого корреляционного ядра $K(x, y)$ - функции на $\mathfrak{X} \times \mathfrak{X}$. Тогда вся информация о точечном процессе содержится в этой единственной функции двух переменных.

Конкретные примеры детерминантных точечных процессов возникают в теории случайных матриц, в некоторых задачах теории представлений, в комбинаторных моделях математической физики и других областях. Замечательно, что корреляционные ядра детерминантных точечных процессов различного происхождения обнаруживают сходную структуру.

Нас будут интересовать предельные переходы для точечных процессов, зависящих от параметра. Например, типичная задача в теории случайных матриц состоит в исследовании предельных свойств спектров случайных матриц порядка $N$, когда параметр $N$ стремится к бесконечности. В моделях точечных процессов, возникающих в теории представлений, предельные переходы могут быть связаны с аппроксимацией бесконечномерной группы конечномерными ([23], [6]).

Язык корреляционных функций хорошо приспособлен к исследованию предельных переходов: сходимость процессов контролируется сходимостью корреляционных функций (подобно тому, как сходимость случайных величин контролируется сходимостью их моментов). Для детерминантных процессов ситуация упрощается, поскольку сходимость корреляционных функций обеспечивается сходимостью корреляционных ядер. В настоящей заметке мы будем исследовать именно предельные переходы для ядер.

Корреляционные ядра $K(x, y)$, возникающие во многих конкретных примеpax из разных областей, имеют следующие общие свойства:

- Пространство $\mathfrak{X}$, на котором определено ядро, является подмножеством B $\mathbb{R}$.

*Работа выполнена при поддержке грантов РФФИ 07-01-91209-ЯФ-а и 08-01-00110-а, а также исследовательского проекта SFB 701 Университета Билефельда. 
- Ядро может быть записано в так называемой интегрируемой форме ${ }^{1)}$, которая в простейшем варианте выглядит так:

$$
K(x, y)=\frac{A(x) B(y)-B(x) A(y)}{x-y},
$$

где $A$ и $B-$ некоторые функции на $\mathfrak{X}$.

В такой ситуации сходимость ядер, зависящих от параметра, часто извлекают из асимптотики функций $A$ и $B$ по параметру. Этот очевидный путь, однако, может приводить к трудоемким вычислениям, если $A$ и $B$ являются достаточно сложными специальными функциями ${ }^{2)}$. В работе обсуждается другой подход, использующий еще одно свойство ядер, которое также выполняется во многих конкретных примерах:

- Оператор $K$ в гильбертовом пространстве $L^{2}(\mathfrak{X})$ (по некоторой естественной мере), задаваемый ядром $K(x, y)$, является проектором. Более того, $K$ можно реализовать в виде спектрального проектора $\mathscr{P}(\Delta)$ для некоторого самосопряженного оператора $D$, действующего в $L^{2}(\mathfrak{X})$. Здесь $\Delta$ - некоторая часть спектра оператора $D$, а сам этот оператор задается разностным или дифференциальным оператором на $\mathfrak{X}$.

При наличии достаточно естественной связи между $K$ и $D$ возникает простая идея: извлекать сходимость корреляционных ядер из сходимости соответствующих самосопряженных операторов $D$. Эта идея была высказана в [10], а затем использована в [13] и [3]. Цель настоящей работы - продемонстрировать эффективность предлагаемого подхода на ряде других примеров. Я рассматриваю лишь достаточно простые примеры, когда точечные процессы живут на одномерной решетке, а не на непрерывном пространстве, и предельный переход не требует скейлинга. Однако я полагаю, что метод может быть полезен и в более сложной ситуации. Некоторый намек на это содержится в разд. 3.4, где объясняется, как можно очень просто угадать скейлинг, приводящий к ядру Эйри.

Структура работы следующая. В 11 напоминаются общие определения, связанные с точечными процессами (подробнее см. [19], [26]). В §2 вводится основная модель: 3-параметрическое семейство вероятностных мер на диаграммах Юнга ${ }^{3)}$. Там же объясняется, как от мер на диаграммах Юнга перейти к точечным процессам на решетке и откуда берется наш основной объект «гипергеометрический» разностный оператор $D$ на решетке. В $\S 3$ обсуждаются предельные переходы, связанные с вырождением z-мер в меру Планшереля. B $\S 4$ исследуется другой предельный режим для Z-мер, приводящий к так называемому гамма-ядру. Это интересное ядро (оно появилось в [7]) описывает асимптотику флуктуаций границы случайной диаграммы Юнга вблизи ее пересечения с диагональю (на другом языке, предельные свойства младших координат Фробениуса).

Я признателен А. М. Бородину за обсуждения. Я благодарен также Р. С. Исмагилову и Ю. А. Неретину за ценные критические замечания.

1) В смысле Итса, Изергина, Корепина и Славнова, см. [11].

2) Например, для решения задачи гармонического анализа на бесконечномерной унитарной группе [6] потребовалось вычислять асимптотику ортогональных многочленов типа Хана, которые выражаются через гипергеометрический ряд ${ }_{3} F_{2}$.

3) Эти меры, называемые z-мерами, были введены в [5] для решения задачи гармонического анализа на бесконечной симметрической группе. См. также обзор [24]. 


\section{§1. Определения}

Пусть $\mathfrak{X}$ - топологическое пространство. Конфигурацией в $\mathfrak{X}$ будем называть подмножество $X \subset \mathfrak{X}$ без точек накопления ${ }^{1)}$. Пространство всех конфигураций в $\mathfrak{X}$ обозначим через $\operatorname{Conf}(\mathfrak{X})$. Если $\mathfrak{X}$ дискретно, то $\operatorname{Conf}(\mathfrak{X})$ есть просто множество $2^{\mathfrak{X}}$ всех подмножеств в $\mathfrak{X}$. Мы будем заниматься вероятностными борелевскими мерами на $\operatorname{Conf}(\mathfrak{X})$. Задание такой меры $P$ позволяет говорить об ансамбле случайных конфигураций, или точечном случайном прочессе.

В дальнейшем пространство $\mathfrak{X}$ будет дискретным, поэтому все последующие определения я приведу для этого простого случая. Относительного общего случая см., например, [19].

Свяжем с $P$ последовательность функций на $\mathfrak{X}, \mathfrak{X} \times \mathfrak{X}, \mathfrak{X} \times \mathfrak{X} \times \mathfrak{X}, \ldots$, называемых корреляционными функииями. По определению $n$-я корреляционная функция $\rho^{(n)}$ получается так: ее значение $\rho^{(n)}\left(x_{1}, \ldots, x_{n}\right)$ на наборе из $n$ точек равно 0 , если среди точек есть совпадающие; в противном случае значение равно вероятности того, что случайная конфигурация $X$ содержит все точки $x_{1}, \ldots, x_{n}$.

Исходная мера $P$ определяется своими корреляционными функциями однозначно.

Точечный процесс $P$ называется детерминантным ([5], [26]), если существует функция $K(x, y)$ на $\mathfrak{X} \times \mathfrak{X}$, такая, что для любого $n=1,2, \ldots$

$$
\rho^{(n)}\left(x_{1}, \ldots, x_{n}\right)=\operatorname{det}\left[K\left(x_{i}, x_{j}\right)\right]_{i, j=1}^{n} .
$$

Будем называть $K(x, y)$ корреляционным ядром процесса $P$. Оператор в координатном гильбертовом пространстве $\ell^{2}(\mathfrak{X})$ с матрицей $[K(x, y)]$ будем называть корреляиионным оператором и обозначать через $K$.

Вся информация о детерминантном точечном процессе $P$ содержится в его корреляционном ядре (эквивалентно, корреляционном операторе).

Простым, но важным примером детерминантных точечных процессов служат ортогональные полиномиальные ансамбли. Приведем определение для интересующего нас дискретного случае (подробнее см. обзор [18]).

Пусть $\mathfrak{X} \subset \mathbb{R}$ - дискретное подмножество с заданной на нем весовой функцией $W(x)>0$. Зафиксируем $N=1,2, \ldots$ По определению ансамбль состоит из $N$-точечных конфигураций $X=\left\{x_{1}, \ldots, x_{N}\right\} \subset \mathfrak{X}$, вероятности которых даются формулой

$$
\operatorname{Prob}(X)=\mathrm{const} \cdot \prod_{i=1}^{N} W\left(x_{i}\right) \cdot \prod_{1 \leqslant i<j \leqslant N}\left(x_{i}-x_{j}\right)^{2} .
$$

где const - подходящий нормирующий множитель.

Известно (см., например, [18]), что такой ансамбль является детерминантным процессом, а его корреляционное ядро $K(x, y)$ следующим образом выражается через ортогональные многочлены $p_{0}=1, p_{1}, p_{2}, \ldots$ с весовой функцией $W(x)$ :

$$
K(x, y)=\sum_{i=0}^{N-1} \widetilde{p}_{i}(x) \widetilde{p}_{i}(y), \quad x, y \in \mathfrak{X},
$$

1) Точнее, то что мы назвали «конфигурацией», следовало бы именовать «простой локально конечной конфигурацией». Простой, потому что исключаются кратные точки, которые допустимы при более общем определении. 
где

$$
\widetilde{p}_{i}(x)=\sqrt{W(x)} \frac{p_{i}(x)}{\left\|p_{i}\right\|_{W}}, \quad x \in \mathfrak{X}, i=0,1, \ldots,
$$

и $\|\cdot\|_{W}$ обозначает норму в весовом гильбертовом пространстве $\ell^{2}(\mathfrak{X}, W)$. Заметим, что функции $\widetilde{p}_{i}(x)$ образуют ортонормированную систему векторов в $\ell^{2}(\mathfrak{X})$. Таким образом, ядро $K(x, y)$ является матрицей конечномерного проектора в $\ell^{2}(\mathfrak{X})$, а именно, проектора на подпространство многочленов степени не выше $N-1$, умноженных на $\sqrt{W(x)}$.

Поскольку $K(x, y)$ равно произведению $\sqrt{W(x) W(y)}$ и $N$-го ядра Кристоффеля-Дарбу для нашей системы ортогональных многочленов, ядро $K(x, y)$ может быть записано в виде

$$
K(x, y)=\mathrm{const} \cdot \frac{\widetilde{p}_{N}(x) \widetilde{p}_{N-1}(y)-\widetilde{p}_{N-1}(x) \widetilde{p}_{N}(y)}{x-y}
$$

и, тем самым, представимо в интегрируемой форме (0.1).

\section{§2. Z-меры и дискретные ортогональные полиномиальные ансамбли}

2.1. Z-меры ([5], [9]). Обозначим через $\mathbb{Y}$ множество всех разбиений $\lambda=$ $\left(\lambda_{1}, \lambda_{2}, \ldots\right)$, которые мы отождествляем с соответствующими диаграммами Юнга. Z-мера с параметрами

$$
z \in \mathbb{C}, \quad z^{\prime} \in \mathbb{C}, \quad \xi \in \mathbb{C} \backslash[1,+\infty)
$$

есть (комплексная) мера $M_{z, z^{\prime}, \xi}$ на множестве $\mathbb{Y}$, которая сопоставляет диаграмме $\lambda \in \mathbb{Y}$ вес

$$
M_{z, z^{\prime}, \xi}(\lambda)=(1-\xi)^{z z^{\prime}} \xi^{|\lambda|}(z)_{\lambda}\left(z^{\prime}\right)_{\lambda}\left(\frac{\operatorname{dim} \lambda}{|\lambda| !}\right)^{2}
$$

Здесь $|\lambda|:=\sum_{i} \lambda_{i}$ (эквивалентно, $|\lambda|$ есть число клеток в диаграмме $\lambda$ );

$$
(z)_{\lambda}=\prod_{i=1}^{\ell(\lambda)}(z-i+1)_{\lambda_{i}}=\prod_{(i, j) \in \lambda}(z+j-i),
$$

где $(a)_{m}=a(a+1) \cdots(a+m-1)$ - символ Похгаммера; $\ell(\lambda)-$ количество ненулевых координат $\lambda_{i}$; второе произведение в (2.2) берется по всем клеткам $(i, j)$ диаграммы $\lambda$, где $i$ и $j$ обозначают номера строки и столбца, в которых находится клетка; наконец, $\operatorname{dim} \lambda$ обозначает количество стандартных таблиц формы $\lambda$ (эквивалентно, размерность неприводимого представления симметрической группы степени $|\lambda|$, индексированного диаграммой $\lambda)$.

Имеет место формула суммирования

$$
\sum_{\lambda \in \mathbb{Y}} M_{z, z^{\prime}, \xi}(\lambda)=1
$$

Здесь мы предполагаем, что либо $|\xi|<1$ (тогда ряд абсолютно сходится), либо параметры $z, z^{\prime}$ являются целыми числами противоположных знаков (тогда ряд обрывается).

Отметим, что z-меры являются частным случаем мер Шура, см. [22]. 
Отметим еще два свойства симметрии z-мер:

$$
M_{z, z^{\prime}, \xi}(\lambda)=M_{z^{\prime}, z, \xi}(\lambda) \quad \text { и } \quad M_{z, z^{\prime}, \xi}\left(\lambda^{\prime}\right)=M_{-z,-z^{\prime}, \xi}(\lambda),
$$

где $\lambda^{\prime}$ обозначает транспонированную диаграмму.

В дальнейшем мы рассматриваем только z-меры с вещественными неотрицательными весами: $M_{z, z^{\prime}, \xi}(\lambda) \geqslant 0$ для всех $\lambda \in \mathbb{Y}$. Эти меры являются вероятностными мерами на $\mathbb{Y}$. Они распадаются на следующие 4 серии.

- Основная серия: Параметры $z, z^{\prime}$ комплексно сопряжены и не вещественны, а параметр $\xi$ - вещественное число и $0<\xi<1$.

- Дополнительная серия: Оба параметра $z, z^{\prime}$ вещественны и содержатся внутри одного и того же открытого интервала вида $(m, m+1)$, где $m \in \mathbb{Z}$, а параметр $\xi$ такой же, как и выше $(0<\xi<1)$.

- Первая вырожденная серия: Один из параметров $z, z^{\prime}$ (скажем, z) является ненулевым целым числом, тогда как другой параметр (тогда это будет $z^{\prime}$ ) есть вещественное число того же знака, причем $\left|z^{\prime}\right|>|z|-1$. Опять-таки, $0<\xi<1$.

- Вторая вырожденная серия: Параметры z и $z^{\prime}$ суть целые числа противоположных знаков. Параметр $\xi$ теперь другой: $\xi<0$.

Если z-мера принадлежит основной или дополнительной серии, то $(z+k)\left(z^{\prime}+\right.$ $k)>0$ для всех целых $k \in \mathbb{Z}$, откуда следует, что веса всех диаграмм строго положительны. Для Z-мер из вырожденных серий некоторые веса обращаются в 0, но все ненулевые веса строго положительны.

\section{2. Подробнее о вырожденных z-мерах. Ансамбли Мейкснера и} Кравчука. Рассмотрим первую вырожденную серию. В силу соотношений симметрии (2.3) достаточно взять $z=N, z^{\prime}=N+c-1$, где $N=1,2, \ldots$ и $c>0$. Тогда вес диаграммы $\lambda$ обращается в нуль в точности при $\ell(\lambda)>N$. Таким образом, носитель Z-меры есть множество диаграмм, содержащихся в горизонтальной полосе ширины $N$. Такие диаграммы $\lambda$ находятся во взаимно однозначном соответствии с $N$-точечными конфигурациями $L$ на $\mathbb{Z}_{+}:=\{0,1,2, \ldots\}$ :

$$
\lambda=\left(\lambda_{1}, \ldots, \lambda_{N}, 0,0, \ldots\right) \longleftrightarrow L=\left(l_{1}, \ldots, l_{N}\right),
$$

где

$$
l_{i}=\lambda_{i}+N-i, \quad 1 \leqslant i \leqslant N .
$$

Нетрудно проверить, что в терминах соответствия $(2.4)$ вес $M_{N, N+c-1, \xi}(\lambda)$ может быть записан в виде (1.1), где надо положить $x_{i}=l_{i}$, а в качестве $W$ взять весовую функцию многочленов Мейкснера [17] на множестве $\mathfrak{X}=\mathbb{Z}_{+}$:

$$
W^{\text {Meixner }}(l)=\frac{(c)_{l} \xi^{l}}{l !}, \quad l \in \mathbb{Z}_{+} .
$$

Таким образом, соответствие $\lambda \rightarrow L$ превращает z-меру первой вырожденной серии с параметрами $z=N, z^{\prime}=N+c-1, \xi$ в $N$-точечный ортогональный полиномиальный ансамбль Мейкснера с параметрами $c$ и $\xi$.

Аналогичный факт имеет место и для второй вырожденной серии. Пусть $z=$ $N$ и $z^{\prime}=-N^{\prime}$, где $N$ и $N^{\prime}$ - два положительных целых числа, и предположим, что $\xi<0$. Тогда вес диаграммы $\lambda$ не обращается в нуль, в точности если $\ell(\lambda) \leqslant N$ и $\ell\left(\lambda^{\prime}\right) \leqslant N^{\prime}$, т. е. $\lambda$ должна содержаться в прямоугольнике $\square \square_{N, N^{\prime}}$ с $N$ строками и $N^{\prime}$ столбцами. Носитель такой меры конечен. 
То же самое соответствие $\lambda \leftrightarrow L$, что в (2.4), задает биекцию между диаграммами $\lambda \subseteq \square_{N, N^{\prime}}$ и $N$-точечными конфигурациями $L$ на конечном множестве $\{0,1, \ldots, \widetilde{N}\}$, где $\widetilde{N}=N+N^{\prime}-1$. Тогда z-мера из второй вырожденной серии превращается в $N$-точечный ортогональный полиномиальный ансамбль на $\mathfrak{X}=\{0,1, \ldots, \widetilde{N}\}$, который задается весовой функцией

$$
W^{\text {Krawtchouk }}(l)=\left(\begin{array}{c}
\widetilde{N} \\
x
\end{array}\right) p^{l}(1-p)^{\widetilde{N}-l}, \quad l=0, \ldots, \widetilde{N},
$$

для ортогональных многочленов Кравчука [17] с параметрами

$$
p:=\frac{\xi}{\xi-1}, \quad \widetilde{N}:=N+N^{\prime}-1 .
$$

Отметим, что условие $\xi<0$ гарантирует, что $0<p<1$.

Итак, ансамбли Мейкснера и Кравчука можно извлечь из z-мер в качестве весьма частного случая. Как мы увидим, полезно использовать и обратный переход:

Тезис 2.1. Z-меры могут быть получены из ансамблей Мейкснера или Кравчука аналитическим продолжением (интерполяиией) по параметрам.

Поясним это высказывание (подробнее см. [9] и [8]). Заметим, что носитель вырожденной меры растет вместе с ростом параметра $N$ (или обоих параметров $N, N^{\prime}$ ), так что в него можно включить любую наперед заданную диаграмму $\lambda \in \mathbb{Y}$. Далее, как видно из (2.1), для любой фиксированной диаграммы $\lambda$ ее вес $M_{z, z^{\prime}, \xi}(\lambda)$ является аналитической функцией от $\xi$, коэффициенты Тейлора которой в $\xi=0$ даются полиномиальными функциями от $z$ и $z^{\prime}$. Это позволяет экстраполировать результаты для вырожденной серии на общий случай, поскольку функции от $z, z^{\prime}$ и $\xi$ с такими свойствами однозначно определяются своим сужением на подмножество, отвечающее параметрам вырожденной серии.

Как мы увидим, тезис 2.1 объясняет происхождение введенного ниже разностного оператора (2.6).

2.3. Гипергеометрический разностный оператор. Рассмотрим теперь основную и дополнительную серии. Мы более не можем использовать соответствие (2.4). Вместо него мы введем другое соответствие, которое будет переводить произвольные разбиения $\lambda \in \mathbb{Y}$ в полубесконечные точечные конфигурации $\underline{X}$ на решетке $\mathbb{Z}^{\prime}=\mathbb{Z}+\frac{1}{2}$ полуцелых чисел. По определению

$$
\lambda \longleftrightarrow \underline{X}=\left\{\lambda_{i}-i+\frac{1}{2} \mid i=1,2, \ldots\right\} .
$$

(Мы называем конфигурацию $\underline{X}$ полубесконечной по той причине, что она содержит все точки решетки, находящиеся достаточно далеко слева от нуля, и не содержит ни одной точки достаточно далеко справа от нуля.)

Между соответствиями (2.4) и (2.5) есть простая связь. Пусть задана диаграмма $\lambda \in \mathbb{Y}$, и пусть $N$ настолько велико, что $N \geqslant \ell(\lambda)$, так что $N$-точечная конфигурация $L$, определенная по (2.4), существует. Тогда $\underline{X}$ получается из $L$ сдвигом влево на величину $N-\frac{1}{2}$ с последующим добавлением бесконечного влево «хвоста» $\left\{\frac{1}{2}-i \mid i=N+1, N+2, \ldots\right\}$. 
Предположим, что параметры $\left(z, z^{\prime}\right)$ лежат в основной или дополнительной серии. Рассмотрим следующий разностный оператор на решетке $\mathbb{Z}^{\prime}$ :

$$
\begin{array}{r}
\mathscr{D}_{z, z^{\prime}, \xi} f(x)=\sqrt{\xi\left(z+x+\frac{1}{2}\right)\left(z^{\prime}+x+\frac{1}{2}\right)} f(x+1)-\left(x+\xi\left(z+z^{\prime}+x\right)\right) f(x) \\
+\sqrt{\xi\left(z+x-\frac{1}{2}\right)\left(z^{\prime}+x-\frac{1}{2}\right)} f(x-1) .
\end{array}
$$

Этот разностный оператор был введен в работах [8] и [9] как результат аналитического продолжения разностного оператора Мейкснера в духе тезиса 2.1. Подробно, процедура аналитического продолжения состоит в следующем:

1. Рассмотрим разностный оператор Мейкснера на $\mathbb{Z}_{+}$:

$$
\mathscr{D}_{c, \xi}^{\text {Meixner }} f(x)=\xi(x+c) f(x+1)-(x+\xi(x+c)) f(x)+x f(x-1) .
$$

Он умножает $n$-й многочлен Мейкснера на $-(1-\xi) n$, см. [17, формула (1.9.5)]. Прибавим к $\mathscr{D}_{c, \xi}^{\text {Meixner }}$ константу $(1-\xi)\left(N-\frac{1}{2}\right)$ и заметим, что первые $N$ многочленов Мейкснера суть в точности собственные векторы получившегося оператора

$$
\mathscr{D}_{c, \xi}^{\text {Meixner }}+(1-\xi)\left(N-\frac{1}{2}\right)
$$

с неотрицательными собственными значениями.

2. Перейдем от весового $\ell^{2}$-пространства $\ell^{2}\left(\mathbb{Z}_{+}, W^{\text {Meixner }}\right)$ к обычному пространству $\ell^{2}\left(\mathbb{Z}_{+}\right)$. Это означает, что (2.7) нужно заменить на композицию операторов

$$
\left(W^{\text {Meixner }}\right)^{1 / 2} \circ\left(\mathscr{D}_{c, \xi}^{\text {Meixner }}+(1-\xi)\left(N-\frac{1}{2}\right)\right) \circ\left(W^{\text {Meixner }}\right)^{-1 / 2} .
$$

3. Сделаем в (2.8) замену переменной $l \rightarrow x:=l-\left(N-\frac{1}{2}\right)$, означающую, что носитель $\mathbb{Z}_{+}$весовой функции преобразуется в подмножество $\left\{-\left(N-\frac{1}{2}\right)+m \mid\right.$ $m=0,1,2, \ldots\} \subset \mathbb{Z}^{\prime}$. Отметим, что это подмножество расширяется по мере роста $N$ и в пределе исчерпывает всю решетку $\mathbb{Z}^{\prime}$.

4. Формально подставим $N=z$ и $c=z^{\prime}-z+1$ в коэффициенты полученного оператора.

Тогда мы получим $\mathscr{D}_{z, z^{\prime}, \xi}$. Таким же образом $\mathscr{D}_{z, z^{\prime}, \xi}$ может быть получен и из разностного оператора, связанного с многочленами Кравчука.

Обозначим через $\left\{e_{x}\right\}, x \in \mathbb{Z}^{\prime}$, естественный ортонормированный базис гильбертова пространства $\ell^{2}\left(\mathbb{Z}^{\prime}\right)$ и через $\ell_{0}^{2}\left(\mathbb{Z}^{\prime}\right) \subset \ell^{2}\left(\mathbb{Z}^{\prime}\right)$ алгебраическое подпространство, состоящее из конечных линейных комбинаций базисных элементов. Мы будем рассматривать $\mathscr{D}_{z, z^{\prime}, \xi}$ (см. (2.6)) как симметрический оператор в $\ell^{2}\left(\mathbb{Z}^{\prime}\right)$ с областью определения $\ell_{0}^{2}\left(\mathbb{Z}^{\prime}\right)$.

Предложение 2.2. Так определенный оператор $\mathscr{D}_{z, z^{\prime}, \xi}$ в существенном самосопряжен.

Доказательство. Стандартный путь состоит в проверке того, что собственные функции разностного оператора (2.6) с невещественными собственными значениями не попадают в $\ell^{2}\left(\mathbb{Z}^{\prime}\right)$. Это можно сделать, явно вычислив собственные функции (они выражаются через гипергеометрическую функцию Гаусса, см. [9]). Вычисления упрощаются, если заменить (2.6) на разностный оператор

$$
\sqrt{\xi}\left|x+\frac{1}{2}\right| f(x+1)-\left(x+\xi\left(z+z^{\prime}+x\right)\right) f(x)+\sqrt{\xi}\left|x-\frac{1}{2}\right| f(x-1),
$$


который отличается от (2.6) на ограниченный оператор (добавление ограниченного самосопряженного оператора сохраняет свойство самосопряженности в существенном).

Я намечу теперь другое рассуждение. Оно, быть может, менее элементарно, но зато не требует вычислений и перебрасывает мостик к представлениям группы $S L(2)$, что оказывается полезным и в других вопросах, связанных с z-мерами.

Рассмотрим комплексную алгебру Ли $\mathfrak{g}_{\mathbb{C}}=\mathfrak{s l}(2, \mathbb{C})$ с базисом

$$
\mathbf{E}=\left[\begin{array}{ll}
0 & 1 \\
0 & 0
\end{array}\right], \quad \mathbf{F}=\left[\begin{array}{ll}
0 & 0 \\
1 & 0
\end{array}\right], \quad \mathbf{H}=\left[\begin{array}{cc}
1 & 0 \\
0 & -1
\end{array}\right]
$$

и коммутационными соотношениями

$$
[\mathbf{H}, \mathbf{E}]=2 \mathbf{E}, \quad[\mathbf{H}, \mathbf{F}]=-2 \mathbf{F}, \quad[\mathbf{E}, \mathbf{F}]=\mathbf{H} .
$$

Вещественная линейная оболочка элементов $\mathbf{E}+\mathbf{F}, i(\mathbf{E}-\mathbf{F})$ и $i \mathbf{H}$ есть вещественная форма $\mathfrak{g} \subset \mathfrak{g}_{\mathbb{C}}:$ это алгебра Ли $\mathfrak{s u}(1,1)$.

Зададим представление $S_{z, z^{\prime}}$ алгебры Ли $\mathfrak{g}_{\mathbb{C}}$ в алгебраическом пространстве $\ell_{0}^{2}\left(\mathbb{Z}^{\prime}\right) \subset \ell^{2}\left(\mathbb{Z}^{\prime}\right)$ следующими формулами для базисных элементов:

$$
\begin{aligned}
& S_{z, z^{\prime}}(\mathbf{E}) e_{x}=\sqrt{\left(z+x+\frac{1}{2}\right)\left(z^{\prime}+x+\frac{1}{2}\right)} e_{x+1}, \\
& S_{z, z^{\prime}}(\mathbf{F}) e_{x}=-\sqrt{\left(z+x-\frac{1}{2}\right)\left(z^{\prime}+x-\frac{1}{2}\right)} e_{x-1}, \\
& S_{z, z^{\prime}}(\mathbf{H}) e_{x}=\left(z+z^{\prime}+2 x\right) e_{x} .
\end{aligned}
$$

Легко проверить, что указанные операторы удовлетворяют выписанным выше коммутационным соотношениям и, тем самым, действительно определяют представление.

Представление $S_{z, z^{\prime}}$ снабжает $\ell_{0}^{2}\left(\mathbb{Z}^{\prime}\right)$ структурой неприводимого модуля Хариш-Чандры над $\mathfrak{g}$, причем эта алгебра действует кососимметрическими операторами. Отсюда следует, что $S_{z, z^{\prime}}$ порождает неприводимое унитарное представление группы $S U(1,1)^{\sim}$, односвязной накрывающей матричной группы $S U(1,1)=S L(2, \mathbb{R})$ (см. [12, с. 14], где доказательство приведено для конкретного примера, но рассуждение носит общий характер). Более того, по известной теореме Хариш-Чандры все векторы из $\ell_{0}^{2}\left(\mathbb{Z}^{\prime}\right)$ суть аналитические векторы этого представления.

С другой стороны, из (2.6) и (2.9) видно, что

$$
\mathscr{D}_{z, z^{\prime}, \xi}=S_{z, z^{\prime}}\left(\sqrt{\xi} \mathbf{E}-\sqrt{\xi} \mathbf{F}-\frac{1+\xi}{2} \mathbf{H}\right)+\frac{1-\xi}{2}\left(z+z^{\prime}\right) \mathbf{1}
$$

где 1 обозначает единичный оператор. Следовательно, все векторы из $\ell_{0}^{2}\left(\mathbb{Z}^{\prime}\right)$ являются аналитическими векторами оператора $\mathscr{D} z, z^{\prime}, \xi$, откуда вытекает ([25, теорема X.39]), что он в существенном самосопряжен.

Замечание 2.3. Использование представления $S_{z, z^{\prime}}$ алгебры Ли $\mathfrak{s l}(2, \mathbb{C})$ подсказано работой Окунькова [21]. Отметим, что соответствующее унитарное представление группы $S U(1,1)^{\sim}$ принадлежит основной или дополнительной серии - в точном соответствии с терминологией для z-мер (которая была введена еще до работы [21], исходя из чисто формальной аналогии). 
Условимся обозначать через $D_{z, z^{\prime}, \xi}$ самосопряженный оператор в $\ell^{2}\left(\mathbb{Z}^{\prime}\right)$, являющийся замыканием оператора $\mathscr{D}_{z, z^{\prime}, \xi}$. Из предложения 2.2 следует, что область определения оператора $D_{z, z^{\prime}, \xi}$ состоит из всех тех функций $f \in \ell^{2}\left(\mathbb{Z}^{\prime}\right)$, которые под действием разностного оператора (2.6) не выводятся из $\ell^{2}\left(\mathbb{Z}^{\prime}\right)$.

Предложение 2.4. Самосопряжсенный оператор $D_{z, z^{\prime}, \xi}$ uмеeт чисто дискретный простой спектр, заполняющий подмножество $(1-\xi) \mathbb{Z}^{\prime} \subset \mathbb{R}$.

Доказательство. Матрица $\sqrt{\xi} \mathbf{E}-\sqrt{\xi} \mathbf{F}-\frac{1+\xi}{2} \mathbf{H}$, фигурирующая в $(2.10)$, сопряжена некоторым элементом из $S U(1,1)$ с матрицей $-\frac{1-\xi}{2} \mathbf{H}$. Стало быть, $D_{z, z^{\prime}, \xi}$ унитарно эквивалентен замыканию оператора $S_{z, z^{\prime}}\left(-\frac{1-\xi}{2} \mathbf{H}\right)+\frac{1-\xi}{2}\left(z+z^{\prime}\right) \mathbf{1}$. Этот оператор является диагональным оператором в базисе $\left\{e_{x}\right\}$, умножающим $e_{x}$ на $-(1-\xi) x$. Это делает утверждение очевидным.

Таким образом, для каждой тройки параметров $\left(z, z^{\prime}, \xi\right)$ из основной или дополнительной серии существует ортонормированный базис $\left\{\psi_{a ; z, z^{\prime}, \xi}\right\}$ в $\ell^{2}\left(\mathbb{Z}^{\prime}\right)$, индексированный точками $a \in \mathbb{Z}^{\prime}$, такой, что

$$
\mathscr{D}_{z, z^{\prime}, \xi} \psi_{a ; z, z^{\prime}, \xi}=(1-\xi) a \psi_{a ; z, z^{\prime}, \xi}, \quad a \in \mathbb{Z}^{\prime} .
$$

Как показано в [9], собственные векторы $\psi_{a ; z, z^{\prime}, \xi}$ можно явно выписать: для них имеется удобное представление через контурный интеграл, а также выражение через гауссовскую гипергеометрическую функцию.

Ввиду связи функций $\psi_{a ; z, z^{\prime}, \xi}$ с гипергеометрической функцией я буду называть $\mathscr{D}_{z, z^{\prime}, \xi}$ гипергеометрическим разностным оператором.

Если $A$ - самосопряженный оператор, у которого 0 не является точкой дискретного спектра, будем обозначать через $\operatorname{Proj}_{+}(A)$ спектральный проектор, отвечающий положительной части спектра оператора $A$. В частности, поскольку спектр оператора $D_{z, z^{\prime}, \xi}$ не содержит 0 , мы вправе образовать проектор $\operatorname{Proj}_{+}\left(D_{z, z^{\prime}, \xi}\right)$.

Образ $z$-меры $M_{z, z^{\prime}, \xi}$ при соответствии $\lambda \mapsto \underline{X}$, введенном в $(2.5)$, задает точечный процесс на $\operatorname{Conf}\left(\mathbb{Z}^{\prime}\right)$, который мы обозначим через $P_{z, z^{\prime}, \xi}$.

Теорема 2.5. $P_{z, z^{\prime}, \xi}$ - детерминантный процесс с корреляиионным ядром $\underline{K}_{z, z^{\prime}, \xi}(x, y)$, которое есть матрица спектрального проектора $\operatorname{Proj}_{+}\left(D_{z, z^{\prime}, \xi}\right)$. To ecmb

$$
\underline{K}_{z, z^{\prime}, \xi}(x, y)=\sum_{a \in \mathbb{Z}_{+}^{\prime}} \psi_{a ; z, z^{\prime}, \xi}(x) \psi_{a ; z, z^{\prime}, \xi}(y), \quad x, y \in \mathbb{Z}^{\prime} .
$$

(Мы не ставим черту над $\psi_{a ; z, z^{\prime}, \xi}(y)$, поскольку собственные функции вещественны, см. формулу (2.1) в [9].)

В приведенной формулировке результат содержится в [9]. Там же даны ссылки на предыдущие работы. Мы называем $K_{z, z^{\prime}, \xi}(x, y)$ дискретным гипергеометрическим ядром. Оно может быть записано и в интегрируемой форме (0.1), см. [9, предложение 3.10].

\section{§3. Мера Планшереля, дискретное ядро Бесселя и дискретное синус-ядро}

3.1. Пуассонизированная мера Планшереля. Рассмотрим предельный режим

$$
\xi \rightarrow 0, \quad z \rightarrow \infty, \quad z^{\prime} \rightarrow \infty, \quad \xi z z^{\prime} \rightarrow \theta
$$


где $\theta>0$ есть новый параметр. Тогда $z$-меры $M_{z, z^{\prime}, \xi}$ сходятся к вероятностной мере $M_{\theta}$ на $\mathbb{Y}$ :

$$
M_{\theta}(\lambda)=\lim _{z z^{\prime} \xi \rightarrow \theta} M_{z, z^{\prime}, \xi}(\lambda)=e^{-\theta} \theta^{|\lambda|}\left(\frac{\operatorname{dim} \lambda}{|\lambda| !}\right)^{2} .
$$

Мера $M_{\theta}$ возникает в результате пуассонизации последовательности мер Планшереля $M^{(n)}, n=1,2, \ldots$, где

$$
M^{(n)}(\lambda)=\frac{(\operatorname{dim} \lambda)^{2}}{n !}, \quad|\lambda|=n .
$$

По этой причине $M_{\theta}$ называется пуассонизированной мерой Планшереля с параметром $\theta$.

Подробнее о мерах Планшереля $M^{(n)}$ см. работы [20] и [27]; см. также [16] и [14]. Пуассонизированная версия $M_{\theta}$ впервые рассматривалась в [1]; дальнейшие результаты получены в [4] и [15].

Как и в 2 , мы используем соответствие $\lambda \mapsto \underline{X}$ для перехода от меры $M_{\theta}$ на $\mathbb{Y}$ к точечному процессу на $\operatorname{Conf}\left(\mathbb{Z}^{\prime}\right)$; обозначим этот точечный процесс через $P_{\theta}$. Он является детерминантным; его корреляционное ядро описано ниже в $\S 3.2$.

3.2. От дискретного гипергеометрического ядра к дискретному ядру Бесселя. В режиме (3.1) формальный предел разностного оператора $\mathscr{D} z, z^{\prime}, \xi$ есть разностный оператор $\mathscr{D}_{\theta}^{\text {Bessel }}$ на решетке $\mathbb{Z}^{\prime}$, действующий по формуле

$$
\mathscr{D}_{\theta}^{\text {Bessel }} f(x)=\sqrt{\theta} f(x+1)-x f(x)+\sqrt{\theta} f(x-1) .
$$

Предложение 3.1. Рассматриваемый как симметрический оператор в $\ell^{2}\left(\mathbb{Z}^{\prime}\right)$ с областью определения $\ell_{0}^{2}\left(\mathbb{Z}^{\prime}\right)$, оператор $\mathscr{D}_{\theta}^{\text {Bessel }}$ является в существенном самосопряженным.

Доказательство. Действительно, $\mathscr{D}_{\theta}^{\mathrm{Bessel}}$ есть сумма диагонального оператора $f \mapsto-x f$ и ограниченного оператора.

Подобно гипергеометрическому оператору (2.6), оператор $\mathscr{D}_{\theta}^{\text {Bessel }}$ связан с некоторым представлением, только другой группы, а именно, универсальной накрывающей $\widetilde{G}$ для группы $G$ движений плоскости $\mathbb{R}^{2}$. Обсудим вкратце эту связь.

Пусть $\mathfrak{g}$ - алгебра Ли группы $G$ и $\mathfrak{g}_{\mathbb{C}}-$ ее комплексификация. В $\mathfrak{g}_{\mathbb{C}}$ есть базис $\{\mathbf{E}, \mathbf{F}, \mathbf{H}\}$ с коммутационными соотношениями

$$
[\mathbf{H}, \mathbf{E}]=\mathbf{E}, \quad[\mathbf{H}, \mathbf{F}]=-\mathbf{F}, \quad[\mathbf{E}, \mathbf{F}]=0 .
$$

Рассмотрим представление $S$ алгебры Ли $\mathfrak{g}_{\mathbb{C}}$ в плотном подпространстве $\ell_{0}^{2}\left(\mathbb{Z}^{\prime}\right)$ $\subset \ell^{2}\left(\mathbb{Z}^{\prime}\right)$, определенное на базисных элементах формулами

$$
S(\mathbf{E}) e_{x}=e_{x+1}, \quad S(\mathbf{F}) e_{x}=-e_{x-1}, \quad S(\mathbf{H}) e_{x}=x e_{x},
$$

Непосредственно проверяется, что все векторы из $\ell_{0}^{2}\left(\mathbb{Z}^{\prime}\right)$ являются аналитическими векторами для $S$, причем $\mathfrak{g}$ действует кососимметрическими операторами. Поэтому $S$ порождает унитарное представление группы $\widetilde{G}$. Справедлива формула

$$
\mathscr{D}_{\theta}^{\text {Bessel }}=S(\sqrt{\theta} \mathbf{E}-\sqrt{\theta} \mathbf{F}-\mathbf{H}),
$$

что является аналогом равенства (2.10). 
Обозначим через $D_{\theta}^{\text {Bessel }}$ замыкание симметрического оператора $\mathscr{D}_{\theta}^{\text {Bessel }}$. Coгласно предложению $3.1, D_{\theta}$ самосопряжен.

Положим

$$
\psi_{a ; \theta}(x):=J_{x+a}(2 \sqrt{\theta}), \quad x \in \mathbb{Z}^{\prime},
$$

где $a \in \mathbb{Z}^{\prime}$ есть параметр, а $J_{m}(\cdot)$ - функция Бесселя индекса $m$.

Предложение 3.2. При любом фиксированном $\theta>0$ функции $\psi_{a ; \theta}$ образуют ортонормированный базис в $\ell^{2}\left(\mathbb{Z}^{\prime}\right)$ u

$$
D_{\theta}^{\text {Bessel }} \psi_{a ; \theta}=a \psi_{a ; \theta}, \quad a \in \mathbb{Z}^{\prime} .
$$

Таким образом, $D_{\theta}^{\text {Bessel }}$ имеет чисто дискретный простой спектр, заполняющий решетку $\mathbb{Z}^{\prime} \subset \mathbb{R}$.

Доказательство. Элемент $\sqrt{\theta} \mathbf{E}-\sqrt{\theta} \mathbf{F}-\mathbf{H} \in i \mathfrak{g}$ сопряжен с $-\mathbf{H}$ относительно присоединенного действия группы $G$. Отсюда следует, что самосопряженные операторы $\overline{S(\sqrt{\theta} \mathbf{E}-\sqrt{\theta} \mathbf{F}-\mathbf{H})}$ и $\overline{S(-\mathbf{H})}$ (здесь черта означает замыкание) сопряжены посредством унитарного оператора, отвечающего некоторому элемен-

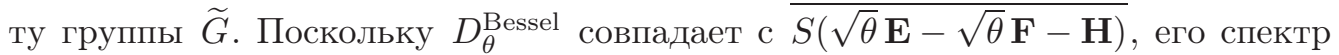
тот же, что у диагонального оператора $\overline{S(-\mathbf{H})}$. Таким образом, мы видим, что спектр действительно такой, как утверждалось.

Тот факт, что функции $\psi_{a ; \theta}(x)$ суть собственные функции оператора $\mathscr{D}_{\theta}^{\text {Bessel }}$, следует из хорошо известных рекуррентных соотношений для функций Бесселя $[2,7.2 .8(56)]$. Наконец, соотношение $\left\|\psi_{a ; \theta}\right\|^{2}=1$ можно доказать таким же образом, как предложение 2.4 в [9], используя контурное интегральное представление функций Бесселя.

Предложение 3.3. В режиме (3.1) операторы $D_{z, z^{\prime}, \xi}$ сходятся $\kappa$ оператору $D_{\theta}^{\text {Bessel }}$ в смысле сильной резольвентной сходимости.

О понятии сильной резольвентной сходимости см. учебник [25, раздел VIII.7].

Доказательство. Очевидно, что $D_{z, z^{\prime}, \xi} \rightarrow D_{\theta}^{\text {Bessel }}$ на подпространстве $\ell_{0}^{2}\left(\mathbb{Z}^{\prime}\right)$. В силу предложений 2.2 и $3.1 \ell_{0}^{2}\left(\mathbb{Z}^{\prime}\right)$ является общей существенной областью для всех рассматриваемых самосопряженных операторов. Тогда утверждение следует из известной общей теоремы [25, теорема VIII.25].

Следствие 3.4. Спектральные проекторь $\operatorname{Proj}_{+}\left(D_{z, z^{\prime}, \xi}\right)$ сильно сходятся $\kappa$ спектральному проектору $\operatorname{Proj}_{+}\left(D_{\theta}^{\text {Bessel }}\right)$.

Доказательство. Это прямое следствие предложения 3.3 и другой общей теоремы, теоремы VIII.24 (b) из [25]. Для применимости этой теоремы важно, что 0 не является точкой дискретного спектра операторов $D_{z, z^{\prime}, \xi}$ и $D_{\theta}^{\mathrm{Bessel}}$, a этот факт следует из описания их спектров, см. предложения 2.4 и 3.2.

Следствие 3.4 показывает, что $\operatorname{Proj}_{+}\left(D_{\theta}^{\text {Bessel }}\right)$ служит корреляционным оператором для $P_{\theta}$. Ядро проектора $\operatorname{Proj}_{+}\left(D_{\theta}^{\text {Bessel }}\right)$ может быть записано в виде

$$
K_{\theta}^{\mathrm{Bessel}}(x, y)=\sum_{a \in \mathbb{Z}_{+}^{\prime}} \psi_{a ; \theta}(x) \psi_{a ; \theta}(y)=\sqrt{\theta} \frac{\psi_{-\frac{1}{2} ; \theta}(x) \psi_{\frac{1}{2} ; \theta}(y)-\psi_{\frac{1}{2} ; \theta}(x) \psi_{-\frac{1}{2} ; \theta}(y)}{x-y} .
$$

Это ядро называется дискретным ядром Бесселя; оно было независимо выведено в [15] и (в несколько иной форме) в [4]. 
3.3. От дискретного ядра Бесселя к дискретному синус-ядру. Рассмотрим следующий предельный режим, зависящий от параметра $c \in(-1,1)$ :

$$
\theta \rightarrow \infty, \quad x \approx 2 c \sqrt{\theta}+\widetilde{x}, \quad \widetilde{x} \in \mathbb{Z},
$$

Это означает, что по мере стремления параметра $\theta$ к $\infty$ мы сдвигаем решетку $\mathbb{Z}^{\prime}$ так, чтобы сфокусироваться на окрестности точки $2 c \sqrt{\theta}$. Имеет место формальный предел

$$
\left(\frac{1}{\sqrt{\theta}} \mathscr{D}_{\theta}^{\text {Bessel }}\right)_{x \rightsquigarrow \tilde{x}} \rightarrow \mathscr{D}_{c}^{\text {sine }}
$$

со следующим разностным оператором в правой части:

$$
\mathscr{D}_{c}^{\text {sine }} f(\widetilde{x})=f(\widetilde{x}+1)-2 c f(\widetilde{x})+f(\widetilde{x}-1), \quad \widetilde{x} \in \mathbb{Z} .
$$

Предложение 3.5. Рассматриваемый как симметрический оператор в $\ell^{2}(\mathbb{Z})$ с областью определения $\ell_{0}^{2}(\mathbb{Z})$, оператор $\mathscr{D}_{c}^{\text {sine } я в л я е т с я ~ в ~ с у щ е с т в е н-~}$ ном самосопряженным.

Его замыкание, которое мы будем обозначать через $D_{c}^{\text {sine }}$, имеет чисто непрерывный спектр кратности 2 , заполняющий интервал $(-2-2 c, 2-2 c)$.

Доказательство. Разностный оператор $\mathscr{D}_{c}^{\text {sine }}$ инвариантен относительно сдвигов решетки $\mathbb{Z}$, что делает его исследование совсем простым. В самом деле, перейдем от решетки $\mathbb{Z}$ к единичной окружности $|\zeta|=1$ посредством преобразования Фурье. Тогда наш оператор превратится в оператор умножения на функцию

$$
\zeta \rightarrow 2(\operatorname{Re} \zeta-c)
$$

откуда легко получается утверждение о спектре.

Предложение 3.6. В предельном режиме (3.2) операторы $D_{\theta}^{\mathrm{Bessel}}$ сходятся $\kappa$ оператору $D_{c}^{\text {sine }}$ в смысле строгой резольвентной сходимости.

Доказательство то же, что в предложении 3.3. Как и в следствии 3.4, получаем отсюда сильную сходимость $\operatorname{Proj}_{+}\left(D_{\theta}^{\text {Bessel }}\right) \rightarrow \operatorname{Proj}_{+}\left(D_{c}^{\text {sine }}\right)$. Ядро проектора $\operatorname{Proj}_{+}\left(D_{c}^{\text {sine }}\right)$ легко вычисляется: оно имеет вид

$$
K_{c}^{\text {sine }}(x, y)=\frac{\sin (\arccos (c / 2)(x-y))}{\pi(x-y)}, \quad x, y \in \mathbb{Z},
$$

и называется дискретным синус-ядром [4].

3.4. От дискретного ядра Бесселя к ядру Эйри. Ядро Эйри впервые возникло в теории случайных матриц: при определенных условиях на матричный ансамбль оно описывает асимптотику спектра «на краю» при стремлении порядка матриц к бесконечности (edge limit regime). Ядро Эйри определено на всей прямой и выражается через классическую функцию Эйри $A i(u)$ и ее производную:

$K^{\operatorname{Airy}}(u, v)=\int_{0}^{+\infty} A i(u+s) A i(v+s) d s=\frac{A i(u) A i^{\prime}(v)-A i^{\prime}(u) A i(v)}{u-v}, \quad u, v \in \mathbb{R}$.

Как было показано в [4] и [15], дискретное ядро Бесселя $K_{\theta}^{\mathrm{Bessel}}(x, y)$ сходится к ядру Эйри $K^{\text {Airy }}(u, v)$, если $\theta \rightarrow+\infty$ и переменная $x \in \mathbb{Z}^{\prime}$ связана с переменной $u \in \mathbb{R}$ скейлингом $x=2 \theta^{1 / 2}+\theta^{1 / 6} \cdot u$. Ниже дается эвристический вывод этого утверждения. 
Пусть $g(u)$ - гладкая функция на $\mathbb{R}$. Сопоставим ей функцию $f(x)$ на $\mathbb{Z}^{\prime}$, полагая $f(x)=g(u)$, где подразумевается, что $x$ и $u$ связаны указанным выше соотношением. Тогда получаем

$$
f(x \pm 1)=g\left(u \pm \theta^{-1 / 6}\right) \approx g(u) \pm \theta^{-1 / 6} \cdot g^{\prime}(u)+\frac{1}{2} \theta^{-1 / 3} \cdot g^{\prime \prime}(u),
$$

так что

$$
\mathscr{D}_{\theta}^{\text {Bessel }} f(x)=\theta^{1 / 2}\left(f(x+1)-x \theta^{-1 / 2} f(x)+f(x-1)\right) \approx \theta^{1 / 6}\left(g^{\prime \prime}(u)-u g(u)\right) .
$$

Это простое вычисление объясняет, как возникает множитель 2 и показатели $1 / 2$ и $1 / 6$.

Далее, уравнение на собственные функции

$$
\mathscr{D}_{\theta}^{\text {Bessel }} \psi=a \psi, \quad a \in \mathbb{Z}^{\prime},
$$

превращается после перенормировки $a=\theta^{1 / 6} s$ в уравнение

$$
\mathscr{D}^{\text {Airy }} \psi=s \psi, \quad s \in \mathbb{R},
$$

где

$$
\mathscr{D}^{\text {Airy }} g(u)=g^{\prime \prime}(u)-u g(u)
$$

- оператор Эйри. Его собственные функции имеют вид

$$
\psi_{s}(u)=A i(u+s), \quad s \in \mathbb{R},
$$

а ядро Эйри отвечает спектральному проектору на положительную часть спектра.

Было бы интересно сделать это рассуждение строгим, используя понятие сходимости по Моско (Mosco convergence).

\section{§4. От дискретного гипергеометрического ядра к гамма-ядру}

Зафиксируем параметры $\left(z, z^{\prime}\right)$ из основной или дополнительной серии и устремим $\xi$ к 1. Как показано в [7], в этом режиме имеется предел для корреляционных ядер. Ниже мы доказываем этот факт по-новому (следствие 4.4).

Из сходимости ядер следует, что образы мер $M_{z, z^{\prime}, \xi}$ при соответствии $(2.5)$ сходятся к некоторой вероятностной мере на $\operatorname{Conf}\left(\mathbb{Z}^{\prime}\right)$, задающей детерминантный точечный процесс. Отметим, что для исходных $z$-мер на $\mathbb{Y}$ картина иная: они сходятся к 0. Такого, конечно, не может произойти на компактном пространстве $\operatorname{Conf}\left(\mathbb{Z}^{\prime}\right)=2^{\mathbb{Z}^{\prime}}$.

Первым указанием на существование предела является то обстоятельство, что гипергеометрический разностный оператор (2.6) сохраняет смысл при формальной подстановке $\xi=1$ : мы получаем тогда разностный оператор

$$
\begin{aligned}
\mathscr{D}_{z, z^{\prime}}^{\text {gamma }} f(x)=\sqrt{\left(z+x+\frac{1}{2}\right)\left(z^{\prime}+x+\frac{1}{2}\right)} f(x+1)-\left(z+z^{\prime}+2 x\right) f(x) \\
+\sqrt{\left(z+x-\frac{1}{2}\right)\left(z^{\prime}+x-\frac{1}{2}\right)} f(x-1) .
\end{aligned}
$$

Далее мы действуем по уже отработанной схеме.

Предложение 4.1. Рассмотрим $\mathscr{D}_{z, z^{\prime}}^{\text {gamma }}$ как симметрический оператор в $\ell^{2}\left(\mathbb{Z}^{\prime}\right)$ с областью определения $\ell_{0}^{2}\left(\mathbb{Z}^{\prime}\right)$. Это дает в существенном самосопряженный оператор. 
Доказательство. Оба рассуждения, приведенные в доказательстве предложения 2.2 , проходят без изменений.

Обозначим через $D_{z, z^{\prime}}^{\text {gamma }}$ самосопряженный оператор, являющийся замыканием оператора $\mathscr{D}_{z, z^{\prime}}^{\text {gamá }}$.

Предложение 4.2. $D_{z, z^{\prime}}^{\text {gama }}$ имеет чисто непрерывный спектр.

Доказательство. Отметим, что при $\xi=1$ соотношение (2.6) превращается в

$$
\mathscr{D}_{z, z^{\prime}}^{\text {gamma }}=S_{z, z^{\prime}}(\mathbf{E}-\mathbf{F}-\mathbf{H}) .
$$

Заметим теперь, что $\mathbf{E}-\mathbf{F}-\mathbf{H}$ есть нильпотентный элемент в $i \mathfrak{s u}(1,1) \subset \mathfrak{s l}(2, \mathbb{C})$. При любом $r>0$ матрица $r(\mathbf{E}-\mathbf{F}-\mathbf{H})$ сопряжена с $\mathbf{E}-\mathbf{F}-\mathbf{H}$ посредством элемента из $S U(1,1)$. Следовательно, $r D_{z, z^{\prime}}^{\text {gamma }}$ унитарно эквивалентен $D_{z, z^{\prime}}^{\text {gamma }}$. Отсюда следует утверждение.

В действительности можно сказать больше: спектр простой и заполняет всю вещественную ось. Это можно вывести, например, из подходящей реализации представления $S_{z, z^{\prime}}$. Собственные функции оператора $D_{z, z^{\prime}}^{\text {gamma }}$, образующие непрерывный спектр, можно явно выписать, см. [8]; они выражаются через классическую функцию Уиттекера.

Предложение 4.3. При $\xi \rightarrow 1$ самосопряженные операторы $D_{z, z^{\prime}, \xi}$ сходятся $\kappa$ самосопряженному оператору $D_{z, z^{\prime}}^{\text {gamma }}$ в смысле строгой резольвентной сходимости.

Доказательство то же, что в предложении 3.3. Так же, как в следствии 3.4, получаем отсюда

Следствие 4.4. При $\xi \rightarrow 1$ проекторы $\operatorname{Proj}_{+}\left(D_{z, z^{\prime}, \xi}\right)$ сильно сходятся $\kappa$ проектору $\operatorname{Proj}_{+}\left(D_{z, z^{\prime}}^{\text {gamma }}\right)$.

Ядро предельного проектора явно описано в [7], [8]. Оно может быть записано в интегрируемой форме $(0.1)$, где функции $A$ и $B$ выражаются через гаммафункцию, откуда проистекает название «гамма-ядро».

\section{ЛитерАтУРА}

[1] J. Baik, P. Deift, K. Johansson, On the distribution of the length of the longest increasing subsequence of random permutations, J. Amer. Math. Soc., 12:4 (1999), 1119-1178.

[2] Г. Бейтмен, А. Эрдейи, Высшие трансцендентные функиии, т. 2, Физматлит, М., 1974.

[3] A. Borodin, V. Gorin, Shuffling algorithm for boxed plane partitions, http://arxiv. org/abs/0804.3071v2.

[4] A. Borodin, A. Okounkov, G. Olshanski, Asymptotics of Plancherel measures for symmetric groups, J. Amer. Math. Soc., 13:3 (2000), 491-515.

[5] A. Borodin, G. Olshanski, Distributions on partitions, point processes and the hypergeometric kernel, Comm. Math. Phys., 211:2 (2000), 335-358.

[6] A. Borodin, G. Olshanski, Harmonic analysis on the infinite-dimensional unitary group and determinantal point processes, Ann. of Math., 161:3 (2005), 1319-1422.

[7] A. Borodin, G. Olshanski, Random partitions and the gamma kernel, Adv. Math., 194:1 (2005), 141-202.

[8] A. Borodin, G. Olshanski, Markov processes on partitions, Probab. Theory Related Fields, 135:1 (2006), 84-152.

[9] A. Borodin, G. Olshanski, Meixner polynomials and random partitions, Moscow Math. J., 6:4 (2006), 629-655. 
[10] A. Borodin, G. Olshanski, Asymptotics of Plancherel-type random partitions, J. Algebra, 313:1 (2007), 40-60.

[11] P. Deift, Integrable operators, in: Differential Operators and Spectral Theory: M. Sh. Birman's 70th anniversary collection, Amer. Math. Soc. Transl., Ser. 2, vol. 189, Amer. Math. Soc., Providence, RI, 1999, 69-84.

[12] J. Dixmier, Représentations intégrables du groupe de De Sitter, Bull. Soc. Math. France, 89 (1961), 9-41.

[13] В. Е. Горин, Непересекающиеся пути и ансамбль ортогональных многочленов Хана, Функц. анализ и его прил., 42:3 (2008), 23-44.

[14] V. Ivanov, G. Olshanski, Kerov's central limit theorem for the Plancherel measure on Young diagrams, in: Symmetric Functions 2001. Surveys of Developments and Perspectives, Proc. NATO Advanced Study Institute (Cambridge, UK, June 25-July 6, 2001), NATO Sci. Ser. II Math. Phys. Chem., vol. 74, Kluwer Acad. Publ., Dordrecht, 2002, 93-151.

[15] K. Johansson, Discrete orthogonal polynomial ensembles and the Plancherel measure, Ann. of Math., 153:1 (2001), 259-296.

[16] S. V. Kerov, Asymptotic representation theory of the symmetric group and its applications in analysis, Transl. of Math. Monographs, vol. 219, Amer. Math. Soc., Providence, RI, 2003.

[17] R. Koekoek, R. F. Swarttouw, The Askey-scheme of hypergeometric orthogonal polynomials and its q-analogue. Delft University of Technology, Faculty of Information Technology and Systems, Department of Technical Mathematics and Informatics, Report no. 98-17, 1998; http://aw.twi.tudelft.nl/ ${ }^{\sim}$ koekoek/askey.html.

[18] W. König, Orthogonal polynomial ensembles in probability theory, Probab. Surv., 2 (2005), 385-447.

[19] A. Lenard, Correlation functions and the uniqueness of the state in classical statistical mechanics, Comm. Math. Phys., 30 (1973), 35-44.

[20] B. F. Logan, L. A. Shepp, A variational problem for random Young tableaux, Adv. Math., 26:2 (1977), 206-222.

[21] A. Okounkov, SL(2) and z-measures, in: Random matrix models and their applications, MSRI Publ., vol. 40, Cambridge Univ. Press, 2001, 71-94; http://arxiv.org/abs/math/0002135.

[22] A. Okounkov, Infinite wedge and random partitions, Selecta Math. (N. S.), 7:1 (2001), 57-81; http://arxiv.org/abs/math/9907127.

[23] G. Olshanski, Point processes related to the infinite symmetric group, in: The Orbit Method in Geometry and Physics: in Honor of A. A. Kirillov, Progr. Math., vol. 213, Birkhäuser, Boston, MA, 2003, 349-393.

[24] G. Olshanski, An introduction to harmonic analysis on the infinite symmetric group, in: Asymptotic Combinatorics with Applications to Mathematical Physics (St. Petersburg, 2001), Lecture Notes in Math., vol. 1815, Springer-Verlag, Berlin, 2003, 127-160.

[25] М. Рид, Б. Саймон, Методы современной математической физики, тт. 1, 2, Мир, М., 1977, 1978.

[26] А. Сошников Детерминантные точечные случайные поля, УМН, 55:5 (2000), 107-160.

[27] А. М. Вершик, С. В. Керов, Асимптотика меры Планшереля симметрической группь и пределъная форма таблии, Юнга, Докл. АН СССР, 233 (1977), 10241027.

Институт проблем передачи информации им. А. А. Харкевича РАН

Поступило в редакцию olsh2007@gmail.com 12 сентября 2008 г. 